\section{OSF Preprints}

Citer cet article : Cheval B, Boisgontier MP (2021) Minimisation de l'effort et autorégulation de l'activité physique. OSF Preprints. doi:10.31219/osf.io/r6fka

Version anglaise de l'article : Cheval B, Boisgontier MP (2021) The theory of effort minimization in physical activity. Exercise and Sport Sciences Reviews. 49(3):168178. doi: $10.1249 / J E S .0000000000000252$

\section{Correspondance :}

Dr. Boris Cheval

Courriel : boris.cheval@unige.ch

Twitter : @chevalboris

Dr. Matthieu P. Boisgontier

Courriel : matthieu.boisgontier@uottawa.ca

Twitter : @MattBoisgontier

\section{Minimisation de l'effort et autorégulation de l'activité physique}

\author{
Boris Cheval ${ }^{1,2}$, Matthieu P. Boisgontier ${ }^{3,4}$ \\ 1 Swiss Center for Affective Sciences, University of Geneva, Geneva, Switzerland \\ ${ }^{2}$ Laboratory for the Study of Emotion Elicitation and Expression (E3Lab), Department of Psy- \\ chology, University of Geneva, Geneva, Switzerland \\ ${ }^{3}$ School of Rehabilitation Sciences, Faculty of Health Sciences, University of Ottawa, Ottawa, \\ Canada \\ ${ }^{4}$ Bruyère Research Institute, Ottawa, Canada
}

\section{Résumé}

La théorie présentée dans cet article vise à expliquer pourquoi de nombreuses personnes ne parviennent pas à transformer leur intention d'être physiquement actif en actions. Les principaux modèles de la motivation humaine indiquent que les processus cognitifs et affectifs sont des déterminants essentiels des comportements de santé, tels que l'activité physique. Cependant, des résultats récents suggèrent qu'un autre processus, l'attrait automatique pour la minimisation de l'effort pourrait permettre de mieux comprendre l'écart entre l'intention d'être actif physiquement et l'engagement réel dans l'activité physique. Les sociétés modernes offrant de nombreuses occasions de réduire la dépense énergétique, surmonter cet attrait automatique pour la minimisation de l'effort s'avère être un défi. La théorie de la minimisation de l'effort en activité physique propose un cadre théorique pour étudier le rapport de force qui régit les interactions entre l'attrait pour la minimisation de l'effort, les ressources cognitives allouées pour y résister et les affects impliqués dans les comportements d'activité physique. Ce cadre théorique a pour objectif de contribuer à une compréhension plus complète et précise des mécanismes neuropsychologiques impliqués dans l'autorégulation de l'activité physique.

\section{Introduction}

Ce matin, vous aviez prévu de faire une promenade, mais comme souvent, vous êtes finalement resté coincé dans votre canapé. Quelles sont les forces susceptibles d'expliquer cet échec à vous engager dans une activité physique planifiée ? S'agitil d'un manque d'attrait pour le comportement à réaliser (aller marcher), d'un trop fort attrait pour le comportement à éviter (rester assis sur votre canapé), ou d'une combinaison de ces deux forces? La théorie de la minimisation de l'effort en activité physique présentée dans cet article vise à répondre à ces questions. L'objectif de cette théorie est de fournir un cadre théorique permettant d'expliquer pourquoi de nombreux individus ayant l'intention d'être actif physiquement ne parviennent pas à concrétiser cette intentions (Rhodes \& Dickau, 2012).

L'implication de processus automatiques dans la régulation des comportements est maintenant largement acceptée (Rhodes, McEwan, \& Rebar, 2019) et commence à être prise en compte dans des contextes cliniques (Boisgontier \& Iversen, 2020). En effet, de nombreuses études testant ces processus automatiques ont montré que les indices (ou signaux) d'activité physique attirent l'attention, déclenchent des réactions affectives positives et produisent des tendances d'approche (Cheval et al., 2020b ; Cheval, Sarrazin, Isoard-Gautheur, Radel, \& Friese, 2015 ; Conroy, Hyde, Doerksen, \& Ribeiro, 2010). Ces résultats suggèrent que des réponses automatiques à des indices d'activité physique qui seraient insuffisamment valorisés (ex.: des réactions affectives négatives ou des tendances à l'évitement) pourraient contribuer à expliquer l'échec de nombreuses personnes à s'engager dans des comportements d'activité physique. Cependant, un fort attrait automatique pour la minimisation de l'effort pourrait être une autre explication. Des études en neuroscience s'intéressant à la prise de décision ont en effet montré que les êtres humains 
favorisaient les comportements qui sont associés à un plus faible effort (Bernacer et al., 2019 ; Klein-Flügge, Kennerley, Friston, \& Bestmann, 2016 ; Prévost, Pessiglione, Météreau, Cléry-Melin, \& Dreher, 2010). Ces résultats ont été confortés par des études en biomécanique et en physiologie qui ont mis en évidence une tendance des êtres humains à recherche le comportement qui présente le meilleur rapport coût/efficacité (Abram, Selinger, \& Donelan, 2019 ; Alexander, 2001 ; Selinger, O'Connor, Wong, \& Donelan, 2015). Malgré ces résultats, la minimisation de l'effort est souvent ignorée dans les études scientifiques testant les déterminants de l'engagement dans l'activité physique. Du fait de ce manque de considération, le rôle potentiel de cet attrait automatique pour la minimisation de l'effort dans la pandémie actuelle d'inactivité physique reste mal compris (Guthold, Stevens, Riley, \& Bull, 2018).

La théorie de la minimisation de l'effort en activité physique inclut dans un même cadre théorique les réactions automatiques aux indices (ou signaux) associés à l'activité physique et l'attrait automatique pour la minimisation de l'effort. Cet attrait automatique est discuté sur la base d'une approche neuropsychologique de l'effort physique (Bernacer et al., 2019 ; Klein-Flügge et al., 2016 ; Prévost et al., 2010) et ancrée dans une perspective évolutionniste (Cheval et al., 2018a ; Lieberman, 2015). Dans la première partie de cet article, nous présentons les théories des comportements d'activité physique basés sur les modèles duaux (Cheval, Sarrazin, \& Radel, 2016). Dans la deuxième partie, nous expliquons comment les humains ont évolué pour éviter les efforts physiques inutiles. Enfin, nous présentons la théorie de la minimisation de l'effort en activité physique.

\section{Modèles duaux des comportements d'activité physique}

Ces dernières années, de nouveaux modèles duaux de l'activité physique ont été développés (Brand \& Ekkekakis, 2018 ; Cheval et al., 2018a ; Conroy \& Berry, 2017). Ces perspectives théoriques sont dites duales parce qu'elles suggèrent que les comportements d'activité physique sont régis non seulement par des processus réfléchis, mais aussi par des processus automatiques. Les processus réfléchis reposent sur des fonctions cérébrales supérieures. Ils sont lents et délibératifs, nécessitent des ressources cognitives et sont élaborés par la pensée consciente. Les processus automatiques reposent sur des associations et des indices heuristiques appris. Ils sont plus rapides et déclenchés de manière non intentionnelle, sollicitent les ressources cognitives dans une moindre mesure et ne nécessitent pas de prise de conscience.

Lorsque les deux systèmes sont convergents et soutiennent l'exécution du même comportement, l'engagement dans l'activité physique est facilité. C'est par exemple le cas lorsqu'une personne est motivée pour être active physiquement et qu'elle a développé des associations affectives positives envers l'activité physique. À l'inverse, lorsque les deux systèmes sont discordants et conduisent à deux comportements opposés, l'engagement dans l'activité physique est entravé. C'est par exemple le cas lorsqu'une personne qui a l'intention d'être active physiquement a développé des associations affectives négatives envers l'activité physique. Dans ce dernier cas, le contrôle de soi et les ressources cognitives détermineront si ce sont les processus automatiques ou les processus réfléchis qui vont être dominants et s'exprimer dans le comportement résultant de cette discordance. Pour mieux comprendre la régulation des comportements d'activité physique, il est donc nécessaire de conceptualiser un cadre théorique qui intègre les (1) processus réfléchis, (2) les processus automatiques ainsi que (3) les ressources de contrôle de soi qui affectent le rapport de force entre ces deux processus.

Les principaux modèles duaux de l'activité physique, à savoir l'évaluation affective automatique de l'activité physique (Conroy \& Berry, 2017) et la théorie affective réflexive de l'inactivité physique et de l'exercice (Brand \& Ekkekakis, 2018), intègrent des processus automatiques qui reposent exclusivement sur les expériences affectives associées à l'activité physique. Plus précisément, ces modèles proposent que la perception d'un indice lié à l'activité physique active automatiquement le concept d'activité physique ainsi que les souvenirs affectifs agréables (ou désagréables) associés à ce concept. Cette activation entraîne une impulsion favorisant la tendance à s'approcher (ou à éviter) l'activité physique. Ainsi, les associations affectives positives sont susceptibles de faciliter l'engagement dans des comportements d'activité physique, tandis que les associations affectives négatives sont susceptibles de l'entraver. D'après ces deux modèles, le déplaisir ressenti lors de l'activité physique pourrait expliquer la difficulté de nombreuses personnes à s'engager dans une activité physique de façon régulière. Conformément à ces théories, des études ont montré que les réponses affectives à l'exercise prédisent l'engagement futur dans les comportements d'activité physique (Rhodes et al., 2019 ; Williams \& Bohlen, 2019 ; Williams et al., 2008; Williams, Dunsiger, Jennings, \& Marcus, 2012)

Cependant, les processus automatiques liés aux expériences affectives associées à une réduction, un arrêt ou une absence d'activité physique (comme par exemple les comportements sédentaires) n'ont jusqu'à présent pas été pris en compte dans l'analyse du comportement d'activité physique. En d'autres termes, la possibilité que le concept de minimisation de l'effort puisse être couplé à des perceptions affectives positives (plaisir) n'a pas été envisagée. Il est pourtant probable que l'évaluation affective automatique positive des indices de minimisation de l'effort puisse influencer notre capacité à changer ou à maintenir un comportement d'activité physique. Des preuves expérimentales récentes soutiennent l'existence d'une telle influence (Bernacer et al., 2019 ; Cheval et al., 2020a ; Cheval et al., 2021 ; Cheval et al., 2018b). Par conséquent, il semble essentiel de prendre en compte la minimisation de l'effort dans les modèles duaux de l'activité physique. 
En résumé, en mettant en évidence le rôle important et jusqu'ici négligé de l'évaluation affective automatique de l'activité physique, les modèles proposés par Brand \& Ekkekakis (2018) et Conroy \& Berry (2017) ont grandement fait progresser la modélisation des processus psychologiques qui sous-tendent les comportements d'activité physique. Ajouter l'évaluation affective automatique d'indices liés à la minimisation de l'effort à cette modélisation devrait permettre d'en améliorer la précision.

\section{2. Évolution et minimisation}

Au cours de l'évolution, la tendance à la minimisation de l'effort se serait développée car elle permettait d'allouer un maximum de ressources énergétiques à l'activité de reproduction et au maintien de l'homéostasie (Gibson \& Mace, 2006 ; Pontzer et al., 2016). Cette tendance se serait donc répandue dans le règne du vivant par le processus de sélection naturelle. Le taux élevé d'inactivité physique dans nos sociétés pourrait donc s'expliquer par ce reliquat de l'évolution qui nous pousserait à éviter les efforts physiques inutiles (Lieberman, 2015).

Cette tendance humaine à minimiser l'effort physique a été largement démontrée dans de multiples domaines, tels que la biomécanique (Abram et al., 2019), la neuroscience (Prévost et al., 2010) et la biologie évolutive (Alexander, 1996). Par exemple, Abram et coll. (2019) ont démontré que les humains optimisaient continuellement leur dépense énergétique pendant la marche, notamment en modulant la vitesse de marche, le balancement des bras, ainsi que la longueur, la largeur et la fréquence des pas. D'autres études, comme celle d'Ivanenko et coll. (2007), ont démontré que le coût énergétique du mouvement déterminait l'adaptation motrice pendant l'apprentissage. Autrement dit, la locomotion humaine s'organise spontanément pour être la moins coûteuse possible en énergie. Dans le domaine des neurosciences, plusieurs études ont démontré que dans les tâches de prise de décision, le cerveau traite l'effort physique comme un coût (Bernacer et al., 2019 ; Klein-Flügge et al., 2016 ; Prévost et al., 2010). Enfin, des données anthropologiques ont rapporté que le quotidien de nos ancêtres, les chasseurs-cueilleurs, consistait en de longues périodes non ambulatoires, suggérant ainsi que l'évolution de l'être humain se soit faite dans un contexte d'inactivité physique importante (Raichlen et al., 2020). La théorie de la minimisation de l'effort en activité physique intègre cet attrait automatique pour la minimisation de l'effort physique dans la modélisation du comportement d'activité physique. Cette approche a pour but de favoriser une compréhension plus complète et plus précise des mécanismes neuropsychologiques impliqués dans l'autorégulation des comportements d'activité physique.

Il est important de noter qu'au cours de l'évolution, les humains ont également développé des capacités d'endurance supérieures aux autres animaux. Ce contraste entre la tendance à conserver l'énergie et la nécessité d'être endurant, qui a abouti à des comportements d'activité physique efficaces et économes, est discuté en détail dans l'article de Cheval and Boisgontier (2021).

\section{La théorie de la minimisation de l'effort en acti- vité physique}

Dans la théorie de la minimisation de l'effort en activité physique, l'attrait automatique pour la minimisation de l'effort physique est conceptualisé comme un processus neuropsychologique favorisant l'exécution et le développement de comportements efficaces et économes. Cette théorie postule que les indices liés au mouvement humain sont perçus comme un effort et que cet effort est traité comme un coût, c'est-à-dire une perception aversive à éviter ou à minimiser. Dans ce cadre, tout mouvement, y compris la respiration, est considéré comme un indice d'effort. Par conséquent, les processus de minimisation de l'effort sont actifs à chaque instant de la vie. Cependant, si cet attrait pour la minimisation de l'effort n'est jamais nul, son intensité varie en fonction des caractéristiques de l'individu, de son comportement et de l'environnement. En d'autres termes, la minimisation de l'effort est une contrainte permanente et dynamique qui influence la régulation du comportement. Lorsqu'un comportement d'activité physique est initié, des indices peuvent déclencher des évaluations automatiques et réfléchies qui soutiennent l'engagement dans ce comportement. Parallèlement, l'effort perçu associé à cet engagement est évalué et interprété comme un coût plus ou moins important. Ces processus aboutissent à des précurseurs du comportement qui vont influencer la décision comportementale (Cheval \& Boisgontier, 2021).

Ici, les comportements d'activité physique sont considérés comme faisant partie d'un continuum énergétique et dépendent de processus réfléchis et automatiques qui peuvent être activés par des indices internes ou externes. L'évaluation positive ou négative de ces indices dépend de leur caractère vital ou non vital et de l'état physiologique de la personne. Une innovation essentielle de la théorie de la minimisation de l'effort en activité physique est l'intégration de l'effort perçu, qui semble essentiel pour une modélisation précise des comportements d'activité physique. En effet, l'évaluation positive ou négative des indices liés au mouvement influence la perception de l'effort qui va à son tour influencer les processus réfléchis (ex. : attitudes raisonnées, intentions explicites) et automatiques (ex. : réactions affectives, tendances d'approche-évitement) menant aux précurseurs comportementaux. Pour que ces précurseurs soutiennent l'engagement dans des comportements associés à une dépense énergétique accrue, les processus automatiques et réfléchis soutenant cet engagement doivent être plus forts que les processus soutenant la minimisation de l'effort. Il est important de noter que le poids relatif des précurseurs réfléchis et des précurseurs automatiques dans le processus décisionnel est modéré par de multiples facteurs tels que l'habitude, la fatigue et la charge cognitive. Chez les personnes qui ont l'intention d'être actives physiquement, on s'attend à ce que des ressources neuropsychologiques 
contrôlées telles que le contrôle de soi aident à surmonter l'évaluation automatique négative de l'effort physique et favorisent l'engagement dans l'activité physique. Cependant, lorsque ces ressources contrôlées font défaut, en raison d'une fatigue importante par exemple, l'influence des processus automatiques, qui comprennent l'attrait automatique pour la minimisation de l'effort, est accrue. La décision comportementale transforme le précurseur comportemental dominant en comportement par la mise en œuvre d'un plan moteur spécifiant la spatialité et la temporalité des contractions musculaires requises pour son exécution.

Pour résumé, les processus automatiques et réfléchis interagissent pour réguler notre comportement. Dans le cas des comportements d'activité physique, s'ajoute notre tendance à minimiser l'effort physique qui entrave notre engagement dans des comportements d'activité physique. Différents facteurs peuvent contribuer à lever ou renforcer cette entrave, tels que le contrôle de soi et la fatigue, respectivement.

\section{Preuves empiriques}

Dans cette partie, nous présentons des preuves empiriques qui soutiennent la théorie de la minimisation de l'effort en activité physique. Cette section ne se veut pas exhaustive, mais vise à présenter les résultats qui nous semblent les plus pertinents pour soutenir la validité de cette théorie.

L'attrait automatique pour la minimisation de l'effort est soutenu par deux études utilisant une tâche d'approche-évitement nommée " tâche du mannequin », dans laquelle les participants sont invités à déplacer un avatar sur l'écran en l'approchant ou l'éloignant de stimuli représentant des comportements sédentaires ou d'activité physique (Cheval et al., 2015 ; Cheval, Sarrazin, \& Pelletier, 2014). Les résultats montrent qu'une tendance d'approche automatique plus élevée vers les stimuli sédentaires (c.-à-d. une plus grande facilité à approcher plutôt qu'à éviter des stimuli sédentaires) et une tendance d'approche plus faible vers les stimuli d'activité physique étaient associées à un engagement plus faible dans une tache de force spontanée (Cheval et al., 2014) et dans des activités physiques modérées à vigoureuses mesurée par accéléromètre (Cheval et al., 2015). Dans cette dernière étude, l'intention d'être physiquement actif prédisait positivement l'activité physique uniquement si la tendance d'approche automatique vers les stimuli sédentaires était faible ou modérée. En d'autres termes, un fort attrait automatique pour des comportements associés à une minimisation de l'effort semblait bloquer l'effet positif de l'intention sur les comportements d'activité physique.

Dans une autre étude, nous avons testé si les comportements minimisant l'effort agissaient comme des tentations (Cheval, Sarrazin, Boisgontier, \& Radel, 2017). Cette étude utilisait la tâche d'amorçage et de décision lexicale de Fishbach, Friedman, and Kruglanski (2003) adaptée au contexte de l'activité physique. Les résultats ont montré que chez les personnes actives physiquement, les mots amorce associés aux comportements sédentaires (ex. : canapé, télévision, ordinateur, assis) facilitaient la reconnaissance des mots liés à l'activité physique (ex. : sport, courir, bouger, effort), tandis que les mots amorce associés à l'activité physique ne facilitaient pas la reconnaissance des mots liés aux comportements sédentaires. Ces résultats sont similaires à ceux rapportés dans le contexte de l'alimentation où les tentations alimentaires (aliments gras et sucrés) activent automatiquement les buts alimentaires (aliments sains) chez les personnes qui réussissent à maintenir leur régime. Autrement dit, ces résultats soutiennent l'hypothèse que les comportements qui minimisent l'effort, tels que les comportements sédentaires, agissent comme des tentations.

L'attrait automatique pour la minimisation de l'effort est également soutenu par des études basées sur l'électroencéphalographie (EEG) (Cheval et al., 2021 ; Cheval et al., 2018b). Dans une première étude, l'activité corticale des participants était enregistrée pendant que les participants réalisaient la tâche du mannequin. Les résultats ont montré qu'éviter les stimuli sédentaires était associé à une activité plus importante du cortex frontal médian et du cortex fronto-central, qui sont en lien avec la résolution de conflits et à l'inhibition, respectivement. Il semble donc que les processus d'inhibition soient essentiels pour contrecarrer notre tendance à éviter les comportements minimisant l'effort. Dans une seconde étude, l'activité corticale des participants était enregistrée pendant qu'ils réalisaient une tâche de go/no-go conçue pour évaluer le contrôle inhibiteur mesuré par le taux d'erreur (réagir de façon erronée à un stimulus « no-go »). Les résultats comportementaux de cette étude ont montré que les erreurs étaient plus nombreuses lorsque les participants devaient éviter de répondre à des stimuli sédentaires que lorsqu'ils devaient éviter de répondre à des stimuli d'activité physique (Cheval et al., 2020a). Ces résultats suggèrent qu'ignorer des stimuli sédentaires nécessite une inhibition plus importante. De plus, les résultats EEG ont montré qu'éviter de répondre aux stimuli sédentaires était associé à une plus forte inhibition dans le cortex fronto-central qu'éviter de répondre aux stimuli d'activité physique (Cheval et al., 2021). En résumé, ces résultats montrent qu'inhiber nos réponses à des stimuli sédentaires nécessite un contrôle inhibiteur plus élevé qu'inhiber nos réponses à des stimuli d'activité physique. Ces résultats suggèrent donc que les ressources cognitives semblent essentielles pour résister à notre attrait automatique pour la minimisation de l'effort.

Ce rôle clé des fonctions cognitives a également été mis souligné par des études longitudinales ayant examiné les associations entre ces fonctions et l'activité physique (Cheval et al., 2020c ; Cheval et al., 2019 ; Daly, McMinn, \& Allan, 2015 ; Sabia et al., 2017). Une étude prospective de 12 ans menée auprès de 105206 adultes âgés de 50 à 90 ans a par exemple montré qu'avoir des ressources cognitives plus élevées est associé à une activité physique plus importante et à une diminution plus lente de 
l'activité physique au cours du vieillissement (Cheval et al., 2020c). Cette étude a également montré que la diminution des ressources cognitives précède le déclin de l'activité physique au cours du vieillissement. Une autre étude a démontré que les fonctions cognitives limitent l'effet de conditions environnementales défavorables sur l'activité physique (Cheval et al., 2019). En somme, ces résultats montrent que les fonctions cognitives sont intimement liées aux comportements d'activité physique et qu'elles semblent nécessaires pour limiter les effets négatifs de l'attrait automatique pour la minimisation de l'effort.

\section{Conclusion}

Bien que l'attrait automatique pour la minimisation de l'effort ait été mis en évidence dans de multiples domaines, son influence sur la régulation des comportements d'activité physique a jusqu'à ce jour été peu prise en compte. La théorie de la minimisation de l'effort en activité physique vise à combler ce manque tout en tirant profit des avancées faites par les précédents modèles.

\section{Remerciements}

BC est soutenu par une bourse Ambizione (PZ00P1_180040) du Fonds national suisse de la recherche scientifique (FNS). MPB est soutenu par une subvention découverte (RGPIN-2021-03153) du Conseil de recherches en sciences naturelles et en génie du Canada (CRSNG) et par une subvention de The Banting Research Foundation.

\section{References}

Abram, S. J., Selinger, J. C., \& Donelan, J. M. (2019). Energy optimization is a major objective in the real-time control of step width in human walking. Journal of Biomechanics, 91, 85-91.

Alexander, R. M. (1996). Optima for animals: Princeton University Press.

Alexander, R. M. (2001). Design by numbers. Nature, 412, 591.

Bernacer, J., Martinez-Valbuena, I., Martinez, M., Pujol, N., Luis, E. O., Ramirez-Castillo, D., \& Pastor, M. A. (2019). An amygdala-cingulate network underpins changes in effort-based decision making after a fitness program. NeuroImage, 203, 116181.

Boisgontier, M. P., \& Iversen, M. D. (2020). Physical inactivity: a behavioral disorder in the physical therapist's scope of practice. Physical Therapy, 100, 743-746.

Brand, R., \& Ekkekakis, P. (2018). Affective-Reflective Theory of physical inactivity and exercise. German Journal of Exercise and Sport Research, 48, 48-58.

Cheval, B., Bacelar, M., Daou, M., Cabral, A., Parma, J., Forestier, C., . . Miller , M. W. (2020a). Higher inhibitory control is required to escape the innate attraction to effort minimization. Psychology of Sport and Exercise, 51, 101781.

Cheval, B., \& Boisgontier, M. P. (2021). The theory of effort minimization in physical activity (TEMPA). Exercise and Sport Sciences Reviews.

Cheval, B., Cabral, D. A. R., Daou, M., Bacelar, M., Parma, J. O., Forestier, C., . . Boisgontier, M. P. (2021). Inhibitory control elicited by physical activity and inactivity stimuli: an EEG study. Motivation Science.
Cheval, B., Miller, M. W., Orsholits, D., Berry, T., Sander, D., \& Boisgontier, M. P. (2020b). Physically active individuals look for more: an eye-tracking study of attentional bias. Psychophysiology, 57, e13582.

Cheval, B., Orsholits, D., Sieber, S., Courvoisier, D. C., Cullati, S., \& Boisgontier, M. P. (2020c). Relationship between decline in cognitive resources and physical activity. Health Psychology, 39, 519-528.

Cheval, B., Radel, R., Neva, J. L., Boyd, L. A., Swinnen, S. P., Sander, D., \& Boisgontier, M. P. (2018a). Behavioral and neural evidence of the rewarding value of exercise behaviors: a systematic review. Sports Medicine, 48, 1389-1404.

Cheval, B., Rebar, A. L., Miller, M. M., Sieber, S., Orsholits, D., Baranyi, G., . . . Boisgontier, M. P. (2019). Cognitive resources moderate the adverse impact of poor neighborhood conditions on physical activity. Preventive Medicine, 126, 105741.

Cheval, B., Sarrazin, P., Boisgontier, M. P., \& Radel, R. (2017). Temptations toward behaviors minimizing energetic costs (BMEC) automatically activate physical activity goals in successful exercisers. Psychology of Sport and Exercise, 30, 110-117.

Cheval, B., Sarrazin, P., Isoard-Gautheur, S., Radel, R., \& Friese, M. (2015). Reflective and impulsive processes explain (in)effectiveness of messages promoting physical activity: a randomized controlled trial. Health Psychology, 34, 10-19.

Cheval, B., Sarrazin, P., \& Pelletier, L. (2014). Impulsive approach tendencies towards physical activity and sedentary behaviors, but not reflective intentions, prospectively predict non-exercise activity thermogenesis. Plos One, 9, e115238.

Cheval, B., Sarrazin, P., \& Radel, R. (2016). Processus automatiques et activités physiques bénéfiques pour la santé. L’Année Psychologique, 116, 295-347.

Cheval, B., Tipura, E., Burra, N., Frossard, J., Chanal, J., Orsholits, D., . . . Boisgontier, M. P. (2018b). Avoiding sedentary behaviors requires more cortical resources than avoiding physical activity: an EEG study. Neuropsychologia, 119, 68-80.

Conroy, D. E., \& Berry, T. R. (2017). Automatic affective evaluations of physical activity. Exercise and Sport Sciences Reviews, 45, 230-237.

Conroy, D. E., Hyde, A. L., Doerksen, S. E., \& Ribeiro, N. F. (2010). Implicit attitudes and explicit motivation prospectively predict physical activity. Annals of Behavioral Medicine, 39, 112-118.

Daly, M., McMinn, D., \& Allan, J. L. (2015). A bidirectional relationship between physical activity and executive function in older adults. Frontiers in Human Neuroscience, 8, 1044.

Fishbach, A., Friedman, R. S., \& Kruglanski, A. W. (2003). Leading us not unto temptation: Momentary allurements elicit overriding goal activation. Journal of Personality and Social Psychology, 84, 296-309.

Gibson, M. A., \& Mace, R. (2006). An energy-saving development initiative increases birth rate and childhood malnutrition in rural Ethiopia. PLoS Med, 3, e87.

Guthold, R., Stevens, G. A., Riley, L. M., \& Bull, F. C. (2018). Worldwide trends in insufficient physical activity from 2001 to 2016: a pooled analysis of 358 population-based surveys with 1.9 million participants. The Lancet Global Health, 6, e1077-1086.

Ivanenko, Y. P., Dominici, N., \& Lacquaniti, F. (2007). Development of independent walking in toddlers. Exercise and Sport Sciences Reviews, 35, 67-73.

Klein-Flügge, M. C., Kennerley, S. W., Friston, K., \& Bestmann, S. (2016). Neural signatures of value comparison in human cingulate cortex during decisions requiring an effort-reward trade-off. Journal of Neuroscience, 36, 10002-10015.

Lieberman, D. E. (2015). Is exercise really medicine? An evolutionary perspective. Current Sports Medicine Reports, 14, 313-319. 
Pontzer, H., Durazo-Arvizu, R., Dugas, L. R., Plange-Rhule, J., Bovet, P., Forrester, T. E., . . Luke, A. (2016). Constrained total energy expenditure and metabolic adaptation to physical activity in adult humans. Current Biology, 26, 410-417.

Prévost, C., Pessiglione, M., Météreau, E., Cléry-Melin, M.-L., \& Dreher, J.C. (2010). Separate valuation subsystems for delay and effort decision costs. Journal of Neuroscience, 30, 14080-14090.

Raichlen, D. A., Pontzer, H., Zderic, T. W., Harris, J. A., Mabulla, A. Z., Hamilton, M. T., \& Wood, B. M. (2020). Sitting, squatting, and the evolutionary biology of human inactivity. Proceedings of the National Academy of Sciences of the United States of America, 117, 7115-7121.

Rhodes, R. E., \& Dickau, L. (2012). Experimental evidence for the intentionbehavior relationship in the physical activity domain: a meta-analysis. Health Psychology, 31, 724-727.

Rhodes, R. E., McEwan, D., \& Rebar, A. L. (2019). Theories of physical activity behaviour change: A history and synthesis of approaches. Psychology of Sport and Exercise, 42, 100-109.

Sabia, S., Dugravot, A., Dartigues, J.-F., Abell, J., Elbaz, A., Kivimäki, M., \& Singh-Manoux, A. (2017). Physical activity, cognitive decline, and risk of dementia: 28 year follow-up of Whitehall II cohort study. British Medical Journal, 357, j2709.

Selinger, J. C., O'Connor, S. M., Wong, J. D., \& Donelan, J. M. (2015). Humans can continuously optimize energetic cost during walking. Current Biology, 25, 2452-2456.

Williams, D. M., \& Bohlen, L. C. (2019). Motivation for exercise: reflective desire versus hedonic dread. In M. H. Anshel, S. J. Petruzzello, \& E. E. Labbé (Eds.), APA Handbook of Sport and Exercise Psychology (Vol. 2, pp. 363-385). Washington, DC: American Psychological Association.

Williams, D. M., Dunsiger, S., Ciccolo, J. T., Lewis, B. A., Albrecht, A. E., \& Marcus, B. H. (2008). Acute affective response to a moderate-intensity exercise stimulus predicts physical activity participation 6 and 12 months later. Psychology of Sport and Exercise, 9, 231-245.

Williams, D. M., Dunsiger, S., Jennings, E. G., \& Marcus, B. H. (2012). Does affective valence during and immediately following a 10-min walk predict concurrent and future physical activity? Annals of Behavioral Medicine, 44, 43-51. 